\title{
GRBS OBSERVED BY MAXI
}

\author{
M. Serino ${ }^{1}$, T. Sakamoto ${ }^{2}$, A. Yoshida ${ }^{2}$, N. Kawai ${ }^{3}$, M. Morii $^{3}$, \\ M. Sugizaki ${ }^{1}$, S. Nakahira ${ }^{4}$, H. Negoro ${ }^{5}$, T. Mihara ${ }^{1}$, Y. Nishimura ${ }^{6}$, \\ Y. Ogawa ${ }^{6}$ and M. Matsuoka ${ }^{1}$
}

\begin{abstract}
Monitor of All-sky X-ray Image (MAXI) on board International Space Station is capable of observing gamma-ray bursts (GRBs) and sending notices of GRBs or other transient events, using real time connection to the ground. MAXI observed 32 GRBs or short X-ray transients as of the end of September 2012. Among them, eleven events were simultaneously detected by other satellites. The observed rate of the MAXI GRBs is about one event per month. This rate is comparable to a past observation with larger effective area and larger field of view. The fact indicates that MAXI has better sensitivity to observe GRBs because of low background. The distribution of the spectral hardness of MAXI GRBs is similar to the results of a past instrument, which is sensitive to similar energy range.
\end{abstract}

\section{Introduction}

Monitor of All Sky Image (MAXI) is an X-ray instrument to monitor the X-ray sky. MAXI is one of the experiments on the Exposed Facility of Japanese Experiment Module (Matsuoka et al. 2009). MAXI consists of two types of cameras, which are Gas Slit Camera (GSC) and Solid-state Slit Camera (SSC). GSC has a relatively

\footnotetext{
${ }^{1}$ MAXI team, Institute of Physical and Chemical Research (RIKEN), 2-1 Hirosawa, Wako, Saitama 351-0198, Japan; e-mail: motoko@crab.riken.jp

${ }^{2}$ Department of Physics and Mathematics, Aoyama Gakuin University, 5-10-1 Fuchinobe, Chuo-ku, Sagamihara, Kanagawa 252-5258, Japan

3 Department of Physics, Tokyo Institute of Technology, 2-12-1 Ookayama, Meguro-ku, Tokyo 152-8551, Japan

${ }^{4}$ ISS Science Project Office, Institute of Space and Astronautical Science (ISAS), Japan Aerospace Exploration Agency (JAXA), 2-1-1 Sengen, Tsukuba, Ibaraki 305-8505, Japan

5 Department of Physics, Nihon University, 1-8-14 Kanda-Surugadai, Chiyoda-ku, Tokyo 101-8308, Japan

6 Department of Applied Physics, University of Miyazaki, 1-1 Gakuen Kibanadai-nishi, Miyazaki, Miyazaki 889-2192, Japan
} 
large effective area and field of view (FOV) than those of SSC. Therefore, GSC is suitable to detect GRBs.

MAXI/GSC has unique capability to observe below $10 \mathrm{keV}$ photons of GRBs. Therefore MAXI/GSC can detect extremely soft GRBs such as X-ray flashes (XRFs; Heise et al. 2001). One of the most comprehensive study of GRBs in this energy range has been accomplished by Sakamoto et al. (2005). They utilized the data sets observed by the Wide-field X-ray Monitor (WXM) (Shirasaki et al. 2003) on the High Energy Transient Explorer 2 (HETE-2; Ricker et al. 2003). Because GSC has a similar energy range to WXM, the GRB samples of WXM/HETE-2 would be the most suitable samples to compare with our GSC samples.

In this paper, we introduce the performance of MAXI including the capability of alert system in Section 2. In Section 3, we show the global properties of the GRBs observed by MAXI using the GSC data. We discuss and summarize our results in Section 4.

\section{Instruments and alert system of MAXI}

\subsection{Performance of MAXI/GSC}

GSC consists of 12 proportional counters with slits and slat collimators (Mihara et al. 2011). Though the total detector area is $5350 \mathrm{~cm}^{2}$, the effective area to a point source is determined by the area of the slit, which is about $10 \mathrm{~cm}^{2}$. GSC looks toward horizontal and zenithal direction. The size of the FOV of MAXI/GSC is 160 degrees by 3 degrees for each direction, which covers $2 \%$ of the whole sky during a certain short period of time ( $\sim 100$ second exposures).

In Table 1 we summarized the performance of MAXI/GSC comparing with HETE-2/WXM. Both instruments utilize position-sensitive proportional counters at the focal plane, but the optic systems differ from each other. Coded mask systems enable instruments to have larger FOV and effective area. On the other hand, slit and collimator optics, which is utilized for GSC, can achieve a low background.

Table 1. Performance of MAXI GSC and HETE-2 WXM.

\begin{tabular}{ccc}
\hline & MAXI GSC & HETE-2 WXM \\
\hline FOV & $160^{\circ} \times 3^{\circ}(2$ directions $)$ & $80^{\circ} \times 80^{\circ}$ \\
Sky coverage & $2 \%$ & $16 \%$ \\
Effective area & $\sim 10 \mathrm{~cm}^{2}$ & $53 \mathrm{~cm}^{2}$ \\
Energy range & $2-20 \mathrm{keV}$ & $2-25 \mathrm{keV}$ \\
Optics & slit and collimator & coded mask \\
\hline
\end{tabular}

\subsection{MAXI transient notice}

MAXI provides notices of GRBs and other transient events through MAXI mailing lists or via GCN. 
There are two types of notices: the automatic notices and the manual notices. The automatic notices can be sent within 10 seconds to a few minutes of the burst time. The position accuracy of this type is about one degree. The manual notices are typically issued within few hours after the burst time. The position accuracy of this type is usually better than 30 arcmin.

\section{Observation and results}

\subsection{Observed rate of MAXI GRBs}

Table 2 shows the list of GRBs observed by MAXI/GSC. The total number of the GRBs is 32 as of the end of September 2012. Eleven GRBs (highlighted with asterisks in the table) are simultaneously detected by other satellites while the two thirds are only observed by MAXI.

Table 2. List of MAXI GRBs.

\begin{tabular}{cl}
\hline year (number) & GRB name \\
\hline $2009(6)$ & GRB 090831*, GRB 090926B*, GRB 091012, GRB 091120*, \\
& GRB 091201, GRB 091230* \\
$2010(11)$ & XRF 100315A, 100327, GRB 100415A, GRB 100510A*, \\
& XRF 100616A, XRF 100701A, GRB 100823A*, 100911, \\
& 101030, XRF 101117A, 101210 \\
$2011(5)$ & GRB 110213B*, 110402, GRB 110426A*, 110916, \\
& GRB 111024A \\
$2012(10)$ & GRB 121027A, GRB 120424A, GRB 120510A*, GRB 120528B, \\
& GRB 120528C, GRB 120614A, GRB 120622A, XRF 120626B, \\
& GRB 120711A*, GRB 120908A* \\
\hline
\end{tabular}

* Simultaneous detection with other satellites.

\subsection{Flux and hardness of MAXI GRBs}

Because the effective area of MAXI/GSC is relatively small comparing with the one of other instruments, most of the MAXI GRBs do not have enough statistics to carry out spectral analyses. Therefore we decided to study two parameters, the average flux and the hardness ratio. The average flux is determined as the total counts of the bursts in the $2-20 \mathrm{keV}$ band divided by the total effective area multiply by the scan duration time around the burst. Here we define the hardness ratio as the flux in the $8-20 \mathrm{keV}$ band divided by the flux in the $2-8 \mathrm{keV}$ band.

We plotted MAXI GRBs in the flux-hardness space (Fig. 1). The bursts only observed by MAXI (triangles) tend to distribute at lower left part of the plot populated by soft and low-flux GRBs. On the other hand, most of the bright ( $>1$ counts $\mathrm{cm}^{-2} \mathrm{~s}^{-1}$ ) bursts, which are also observed by other instruments (circles), are relatively hard. From this figure, we conclude MAXI/GSC is sensitive to soft and dim bursts. 


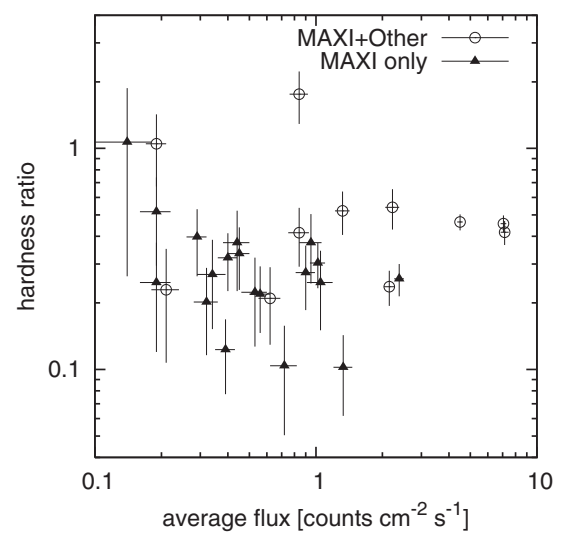

Fig. 1. Flux and hardness of MAXI GRBs. The bursts which are also observed by other instruments are plotted with circles. The bursts which are not observed by other instruments are plotted with triangles.

In order to compare the results with HETE-2/WXM, we plotted histograms of flux and hardness (Fig. 2). From the left panels, we can see that the average flux of the MAXI bursts are systematically lower than that of the WXM GRBs. This results confirm that the MAXI/GSC is more sensitive to weak GRBs than HETE-2/WXM. In the hardness distribution (right panels), there is no significant difference between the MAXI/GSC and the HETE-2/WXM samples. In Sakamoto et al. (2005), about one third of GRBs is classified into XRFs. Although it is difficult to classify GRBs solely from the MAXI data, we expect that roughly one third of the MAXI GRBs may be classified into XRFs.
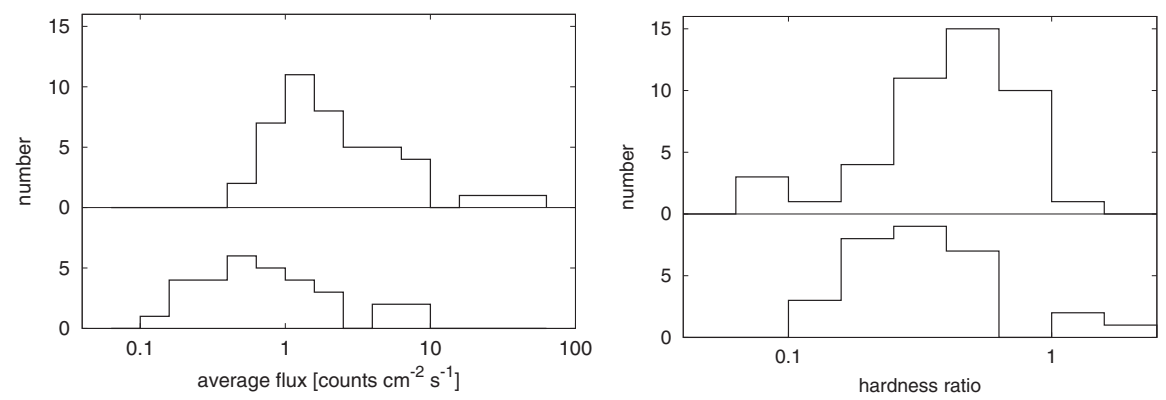

Fig. 2. Histograms of flux (left) and hardness (right) distribution of MAXI and HETE GRBs. The histograms of top panels are result of HETE-2/WXM (Sakamoto et al. 2005). The histograms of MAXI/GSC are plotted in the bottom panels. 


\section{Discussions and conclusion}

MAXI observed 32 GRBs or short X-ray transients as of the end of September 2012. The observed rate is about once a month. The one third of the observed events are also detected by other instruments. Although MAXI/GSC has smaller sky coverage and smaller effective area, this rate is comparable to that of HETE-2/WXM. This fact suggests that MAXI/GSC is detecting dimmer bursts than HETE-2/WXM. Indeed the flux distribution of the MAXI/GSC bursts (Fig. 2) supports this hypothesis.

MAXI has unique capability to observe low-energy portion of GRBs below $10 \mathrm{keV}$. We showed that hardness distribution of MAXI GRBs is similar to that of HETE-2/WXM, which means about one third of them are expected to be XRFs.

Finally, we would like to emphasize that follow-up observations are needed to reveal the nature of XRFs. We strongly encourage follow-up observers to observe afterglows of MAXI XRFs.

\section{References}

Heise, J., Zand, J.I., Kippen, R.M., et al., 2001, in "Gamma-ray Bursts in the Afterglow Era", ed. E. Costa, F. Frontera \& J. Hjorth, p. 16

Matsuoka, M., Kawasaki, K., Ueno, S., et al., 2009, PASJ, 61, 999

Mihara, T., Nakajima, M., Sugizaki, M., et al., 2011, PASJ, 63, S623

Ricker, G.R., Atteia, J.-L., Crew, G.B., et al., 2003, in "Gamma-Ray Burst and Afterglow Astronomy 2001: A Workshop Celebrating the First Year of the HETE Mission", ed. G.R. Ricker \& R.K. Vanderspek, p. 3

Sakamoto, T., Lamb, D.Q., Kawai, N., et al., 2005, ApJ, 629, 311

Shirasaki, Y., Kawai, N., Yoshida, A., et al., 2003, PASJ 55, 1033

Sugizaki, M., Mihara, T., Serino, M., et al., 2011, PASJ, 63, S635 
